\title{
Nomogram Incorporating Multimodal Transvaginal Ultrasound Assessment at 20 to 24 Weeks' Gestation for Predicting Spontaneous Preterm Delivery in Low-Risk Women
}

\author{
Lingli Jiang',*, Lei Peng',*, Miaoling Rong², Xiaozhi Liu³, Qinxia Pang', Huaping Li', Ying Wang', \\ Zhou Liu (D)' \\ 'Department of Obstetrics and Gynecology, Shanghai University of Medicine \& Health Sciences Affiliated Zhoupu Hospital, Shanghai, People's \\ Republic of China; ${ }^{2}$ Department of Obstetrics and Gynecology, First Maternity and Infant Hospital Affiliated to Tongji University, Shanghai, People's \\ Republic of China; ${ }^{3}$ Department of Ultrasound, Shanghai University of Medicine \& Health Sciences Affiliated Zhoupu Hospital, Shanghai, People's \\ Republic of China \\ *These authors contributed equally to this work
}

Correspondence: Zhou Liu, Department of Obstetrics and Gynecology, Shanghai University of Medicine \& Health Sciences Affiliated Zhoupu Hospital, No. 1500, Zhouyuan Road, Pudong New Area, Shanghai, 2013I8, People's Republic of China, Tel +86 I8930837705, Email zp_liuz@sumhs.edu.cn

Background: The majority of women who experience spontaneous preterm delivery (SPTD) have low-risk, asymptomatic pregnancies with a cervical length $(C L) \geq 25 \mathrm{~mm}$ and no clear risk factors. Despite the fact that cervical elastography is a potential tool for predicting SPTD, there is currently no feasible solution to make a reliable prediction for preventing SPTD.

Objective: The aim of this study was to construct a nomogram including multimodal transvaginal ultrasound parameters during the second trimester to predict SPTD in low-risk women.

Methods: This multi-center study enrolled 1260 women with singleton pregnancies between 20 and 24 weeks' gestation. CL and cervical elastography data were obtained when they were undergoing the second-trimester anomaly scan. Univariate and multivariate Logistic regression were utilized to screen predictors independently related to SPTD from the maternal characteristics and multimodal ultrasound data. Then construct a nomogram to determine the likelihood of SPTD in pregnant women.

Results: A total of 66 pregnancies in the training cohort $(7.8 \%, 66 / 842)$ and 37 pregnancies $(8.9 \%, 37 / 418)$ in the validation cohort ended in SPTD. Age, uterine curettage, CL, and strain in the anterior lip of internal os were the independent predictors of SPTD $(P<0.001,<0.001$, $=0.007$, and $<0.001$, respectively). These predictors constituted a nomogram to predict the probability of SPTD for a pregnant woman in her second trimester. It showed good discrimination $(\mathrm{C}$-index $=0.898$ and 0.839$)$, calibration $(P=0.258$ and 0.115$)$, and yielded net benefits both in the training and validation cohorts.

Conclusion: The nomogram including data of multimodal transvaginal ultrasound at 20 to 24 weeks' gestation is expected to identify women with SPTD in the low-risk, asymptomatic population.

Keywords: cervical length measurement, elasticity imaging techniques, nomograms, pregnancy trimester, second, premature birth

\section{Introduction}

One of the leading causes of under-5 deaths was preterm birth complication. ${ }^{1}$ Cervical insufficiency is an important cause of preterm birth although many risk factors are not fully understood. ${ }^{2}$ Currently, transvaginal ultrasound measurement of cervical length (CL) is mainly recommended to predict the risk of spontaneous preterm delivery (SPTD). ${ }^{3}$ However, when using the $25 \mathrm{~mm}$ cut-off value in the second trimester, the sensitivity for predicting SPTD was only $37.3 \%{ }^{4}$ This means that this alone may not be able to detect a considerable proportion of women who will subsequently have an 
SPTD. ${ }^{5,6}$ Furthermore, more than half of SPTD cases occur in low-risk pregnancies, and the majority have no obvious risk factors. ${ }^{7}$

The assessment of changes in cervical stiffness with cervical elastography is proved to help predict SPTD because cervical softening at inappropriate times seems to be related to preterm birth. ${ }^{8}$ Several studies have evaluated this promising technology and found that cervical elastography showed better performance in predicting SPTD than CL. ${ }^{9-12}$ Recent research further revealed that cervical elastography combined with CL obtained a superior performance in SPTD prediction. ${ }^{9}{ }^{13}$ However, while this combination enhances prediction accuracy, it is difficult to make a credible prediction based on measured CL and cervical elasticity in order to carry out corresponding interventions, limiting its use in clinical diagnosis and prevention. Nomogram created by regression analysis is a graphical display tool that facilitates calculation and interpretation of prediction results, and is of great significance for optimizing individualized treatment. It has been widely employed to predict the clinical outcomes of diverse cancers. ${ }^{14-16}$

Therefore, the current study aimed to develop a nomogram including the multimodal transvaginal ultrasound assessment to predict SPTD in asymptomatic and low-risk women presenting for a second-trimester anomaly scan in the interest of precision medicine. The performance of the nomogram was validated by discrimination, calibration, and decision curve analysis (DCA).

\section{Materials and Methods}

The institutional review board of Shanghai Pudong New District Zhoupu Hospital approved this study (2021-C-146) and informed consents were obtained from all participants. It was conducted following the Declaration of Helsinki.

\section{Study Participants}

From January 2019 to May 2021, participating in this multi-center study were 1260 consecutively enrolled women with singleton pregnancies between 20 and 24 weeks' gestation, who underwent the second-trimester anomaly scan in Shanghai Pudong New District Zhoupu Hospital (enrolled for training cohort) and Shanghai First Maternity and Infant Hospital (enrolled for validation cohort). The included low-risk women were defined as those aged 18 to 45 years, with a $C L \geq 25 \mathrm{~mm}$, no history of preterm birth and cervical surgery, and no genital malformation. The following were the criteria for exclusion: 1) tocolytic or cerclage-assisted pregnancies, 2) recognized causes of preterm delivery (premature rupture of membranes, multiple pregnancies, etc.), and 3) elective terminations and indicated preterm deliveries. Only cases of SPTD occurred by spontaneous onset of labor or spontaneous rupture of membranes before 37 weeks' gestation were evaluated. Maternal characteristics including age, obstetrical history, history of uterine curettage, prepregnancy body mass index (BMI), smoking status, in vitro fertilization and embryo transfer (IVF-ET), weeks of gestation at the examination, CL, and strain of distinct regions of interest (ROIs) in the cervix were collected.

\section{$\mathrm{CL}$ and Elastographic Measurements}

For CL measurement and cervical elastography, a transvaginal ultrasound examination was performed utilizing the GE Voluson E10 Ultrasound System (GE Medical Systems, Zipf, Austria) with a transvaginal probe. The interobserver reliability was not evaluated because only one sonographer in each center completed the assessment to minimize the interruption in the busy clinical practice and avoid prolonged examinations. A sagittal view of the cervix was acquired by inserting the probe into the anterior fornix of the vagina. CL was determined as the distance between the internal and external cervical os without pressure on the cervix.

Elastography was done after the CL was measured by applying external pressure to the anterior lip of the cervix with gentle and rhythmic motions mostly perpendicular to the longitudinal axis until the posterior lip was dislocated. Under the premise of the green elastic scale, which was designed for the performance of strain image acquisition, tissue deformation was calculated as strain [0 (hard) to 1 (soft)] and shown on a colored scale from blue to red indicating stiff to soft tissue. We chose four ROIs in the cervix near the anterior lip of internal os (AI), anterior lip of external os (AE), posterior lip of internal os (PI), and posterior lip of external os (PE), respectively to determine the average strain inside each ROI (Figure 1). 


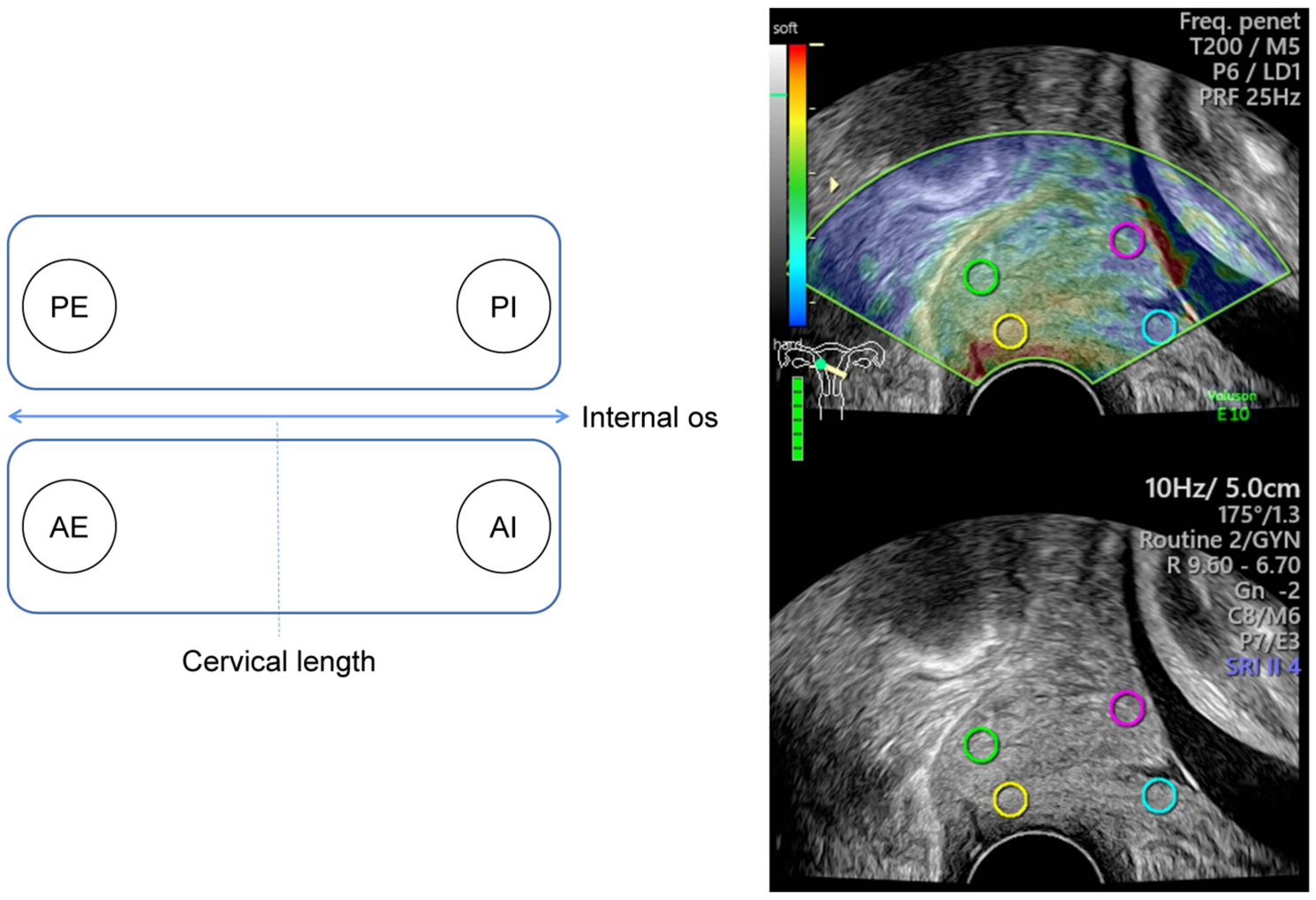

Figure I Schematic representation of four ROI settings (Al: anterior lip of internal os, AE: anterior lip of external os, PI: posterior lip of internal os, and PE: posterior lip of external os) of cervical elastography under the sagittal view of vaginal ultrasound assessment.

Abbreviation: ROI, region of interest.

\section{Nomogram Construction}

In the training cohort, univariate and multivariate Logistic regression were first performed to investigate the predictors independently related to SPTD. Based on these factors, a nomogram was then constructed to determine the likelihood of SPTD in women with singleton pregnancy between 20 and 24 weeks' gestation. The performance of nomogram was verified both in the training and validation cohorts.

\section{Statistical Analysis}

The sample size estimation was performed using an online sample size calculators (https://sample-size.net/sample-size-confinterval-proportion/). The sample size was appropriate based on the result of sample size calculation (expected proportion: 0.1 , total width of confidence interval: 0.05 , and confidence level: $95 \%$ ). The normal distributed, skewed distributed, and categorical data were compared using independent sample $t$-test, Mann-Whitney $U$-test, and Chi-square test, respectively. The multivariate Logistic regression model only included variables with a $P<0.05$ in the univariate Logistic regression analysis, and calculated the odds ratio (OR) and $95 \%$ confidence interval (CI) of each independent factor to serve as the components of the newly established nomogram. In the verification of the nomogram, bootstrap resampling (1000 times) analysis was applied for verification. The discrimination and calibration of the nomogram were evaluated by $\mathrm{C}$-index and Hosmer-Lemeshow (HL) tests, respectively. Their distribution were displayed through the receiver operating characteristic (ROC) curve and the calibration curve. DCA was utilized to evaluate the clinical net benefits. IBM SPSS Statistics 22.0 (IBM Corp., Armonk, NY, USA) and R package version 3.6.2 were used to conduct the statistical analyses.

\section{Results}

In this study, 66 pregnancies in the training cohort $(7.8 \%, 66 / 842)$ and 37 pregnancies $(8.9 \%, 37 / 418)$ in the validation cohort ended in SPTD. Table 1 summarizes the clinical characteristics of the two cohorts. Among them, uterine curettage 
Table I Clinical Characteristics of Pregnant Women in the Training and Validation Cohorts

\begin{tabular}{|c|c|c|c|c|}
\hline \multicolumn{2}{|l|}{ Variable } & Training Cohort $(n=842)$ & Validation Cohort $(n=4 \mid 8)$ & $P$ value \\
\hline \multirow{2}{*}{\multicolumn{2}{|c|}{$\begin{array}{l}\text { Age, years } \\
\text { Multipara, n(\%) }\end{array}$}} & $28(25,3 \mathrm{I})$ & $29(25,32)$ & $0.271^{\#}$ \\
\hline & & $302(35.9 \%)$ & $159(38.0 \%)$ & $0.451^{\$}$ \\
\hline \multirow[t]{4}{*}{ Uterine curettage } & 0 & $549(65.2 \%)$ & 267 (63.8\%) & $0.160^{\$}$ \\
\hline & I & $244(29.0 \%)$ & 137 (32.8\%) & \\
\hline & 2 & $38(4.5 \%)$ & $12(2.9 \%)$ & \\
\hline & $\geq 3$ & II (I.3\%) & $2(0.5 \%)$ & \\
\hline \multicolumn{2}{|c|}{ Prepregnancy BMI, kg/m² } & $22.51 \pm 3.84$ & $21.42 \pm 3.37$ & $0.092 *$ \\
\hline \multicolumn{2}{|c|}{ Smoking, $n(\%)$} & $82(9.7 \%)$ & 34 (8.1\%) & $0.354^{\$}$ \\
\hline \multicolumn{2}{|l|}{ IVF-ET, n(\%) } & $116(13.8 \%)$ & $74(17.7 \%)$ & $0.067^{\$}$ \\
\hline \multicolumn{2}{|c|}{ Weeks of gestation at the examination, weeks } & $22(21,23)$ & $22(21,23)$ & $0.081^{\#}$ \\
\hline \multicolumn{2}{|c|}{$\mathrm{CL}, \mathrm{mm}$} & $36(33,39)$ & $35(32,37)$ & $0.072^{\#}$ \\
\hline \multirow[t]{4}{*}{ Strain in the ROI } & Al & $0.23(0.18,0.27)$ & $0.23(0.19,0.28)$ & $0.099^{\#}$ \\
\hline & $\mathrm{PI}$ & $0.25(0.20,0.3 \mathrm{I})$ & $0.26(0.20,0.32)$ & $0.136^{\#}$ \\
\hline & $\mathrm{AE}$ & $0.35(0.29,0.42)$ & $0.33(0.27,0.39)$ & $0.283^{\#}$ \\
\hline & $\mathrm{PE}$ & $0.38(0.3 \mathrm{I}, 0.46)$ & $0.39(0.32,0.47)$ & $0.236^{\#}$ \\
\hline
\end{tabular}

Note: *For independent sample $t$-test, ${ }^{\$}$ for chi-square test, and ${ }^{\#}$ for Mann-Whitney U-test.

Abbreviations: BMI, body mass index; IVF-ET, in vitro fertilization and embryo transfer; CL, cervical length; Al, anterior lip of internal os; AE, anterior lip of external os; PI, posterior lip of internal os; PE, posterior lip of external os.

was found in $293(34.8 \%)$ and $151(36.2 \%)$ women in the training and validation cohorts, respectively. For all characteristics, there were no significant differences between the two cohorts (all $P$ values $>0.05$ ).

\section{Independent Predictors for SPTD}

Table 2 reports the univariate and multivariate Logistic analyses performed on the training cohort to determine the relationship between potential variables and SPTD. On univariate analysis, age, uterine curettage, smoking, IVF-ET, CL, and strain of AI and PI were associated with SPTD $(P<0.001,<0.001,=0.027,=0.039,<0.001$, and $<0.001$, respectively). The multivariate analysis revealed that age, uterine curettage, $\mathrm{CL}$, and strain of AI were the independent predictors of SPTD $(P<0.001,<0.001,=0.007$, and $<0.001$, respectively $)$.

\section{Nomogram Establishment}

Based on the independent predictors, a nomogram was established to predict the probability of SPTD for a pregnant woman in her second trimester (Figure 2). To estimate the probability, each predictor was given a number with a weight equal to the OR value. For example, a 35-year-old pregnant woman received a uterine curettage. Her CL and strain of AI were $30 \mathrm{~mm}$ and 0.3 , respectively. The total score of the nomogram was about 180 , which indicated that the risk of SPTD was approximately $60 \%$.

\section{Nomogram Verification}

In the verification based on the training cohort, the overfitting-corrected C-index obtained after 1000 bootstrapping was (0.898, 95\% CI: 0.876-0.918), indicating good discrimination. The calibration test revealed no significant difference between the predicted and actual SPTD probability (HL test: $\chi^{2}=10.103, P=0.258$ ), confirming good calibration. The ROC curve and calibration curve for evaluating the discrimination and calibration of the nomogram are plotted in Figure 3A and B. The DCA curve showed that the nomogram yielded clinical net benefit (Figure 3C). In the verification based on the validation cohort from another center, the nomogram also showed good discrimination $(0.839,95 \% \mathrm{CI}$ : 0.800-0.873) (Figure 3D) and good calibration (HL test: $\chi^{2}=13.492, P=0.115$ ). However, the calibration plot revealed that the nomograms might underestimate the likelihood of SPTD when the predicted probability was $>20 \%$ (Figure 3E). The DCA plot still yielded net benefits although it was lower than that based on the training cohort (Figure 3F). 
Table 2 Univariate and Multivariate Logistic Analyses to Determine the Independent Predictors Associated with SPTD

\begin{tabular}{|c|c|c|c|c|c|c|c|}
\hline \multicolumn{2}{|l|}{ Variable } & \multicolumn{3}{|c|}{ Univariate Logistic Regression } & \multicolumn{3}{|c|}{ Multivariate Logistic Regression } \\
\hline & & $P$ value & OR & $95 \% \mathrm{Cl}$ & $P$ value & OR & $95 \% \mathrm{Cl}$ \\
\hline \multirow{2}{*}{\multicolumn{2}{|c|}{$\begin{array}{l}\text { Age } \\
\text { Multipara }\end{array}$}} & $<0.001$ & 1.271 & $1.198-1.349$ & $<0.001$ & 1.287 & $1.181-1.402$ \\
\hline & & 0.251 & 1.593 & $0.828-3.066$ & & & \\
\hline \multirow[t]{4}{*}{ Uterine curettage } & 0 & \multicolumn{3}{|c|}{ Reference } & \multicolumn{3}{|c|}{ Reference } \\
\hline & 1 & 0.014 & 2.154 & $1.168-3.971$ & 0.239 & 1.613 & $0.727-3.580$ \\
\hline & 2 & $<0.001$ & $|3.34|$ & $6.114-29.109$ & $<0.001$ & 8.493 & $2.7 \mid 2-26.602$ \\
\hline & $\geq 3$ & $<0.001$ & 60.986 & $15.174-245.102$ & $<0.001$ & 103.642 & $12.116-886.560$ \\
\hline \multirow{5}{*}{\multicolumn{2}{|c|}{$\begin{array}{l}\text { Prepregnancy BMI, } \\
\text { Smoking } \\
\text { IVF-ET } \\
\text { Weeks of gestation at } \\
\text { examination } \\
\text { CL }\end{array}$}} & 0.168 & 1.204 & $0.626-2.317$ & & & \\
\hline & & 0.027 & 1.834 & $1.354-2.515$ & 0.162 & 1.648 & $0.952-2.853$ \\
\hline & & 0.039 & 1.473 & $1.037-2.092$ & 0.203 & 1.253 & $0.724-2.169$ \\
\hline & & 0.710 & 0.967 & $0.810-1.154$ & & & \\
\hline & & $<0.001$ & 0.864 & $0.808-0.924$ & 0.007 & 0.880 & $0.80 \mathrm{I}-0.966$ \\
\hline \multirow[t]{4}{*}{ Strain in the cervix } & $\mathrm{Al}$ & $<0.001$ & $1.256^{*}$ & $1.201-1.303$ & $<0.001$ & $1.257^{*}$ & $1.190-1.327$ \\
\hline & $\mathrm{PI}$ & 0.023 & $1.164 *$ & $1.103-1.219$ & 0.105 & $1.063^{*}$ & $0.940-1.203$ \\
\hline & $\mathrm{AE}$ & 0.138 & І.083* & $0.858-1.367$ & & & \\
\hline & $\mathrm{PE}$ & 0.174 & $1.107 *$ & $0.877-1.398$ & & & \\
\hline
\end{tabular}

Note: *Denotes a specific $O R$ value, indicating that the risk increases by 0.01 unit increments.

Abbreviations: SPTD, spontaneous preterm delivery; BMI, body mass index; IVF-ET, in vitro fertilization and embryo transfer; CL, cervical length; Al, anterior lip of internal os; $\mathrm{AE}$, anterior lip of external os; PI, posterior lip of internal os; PE, posterior lip of external os.

\section{Discussion}

Accurate prediction of SPTD in low-risk women in their second trimester may allow for more effective management to avoid preterm birth. To solve this issue, we developed a nomogram incorporating age, history of uterine curettage, CL, and strain of AI to predict SPTD in asymptomatic and low-risk women at 20 to 24 weeks' gestation. It showed good performance and yielded net benefits both in the training and validation cohorts, suggesting that it might become an SPTD screening tool for low-risk pregnant women.

Points

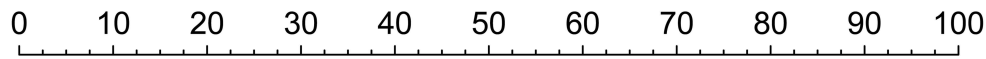

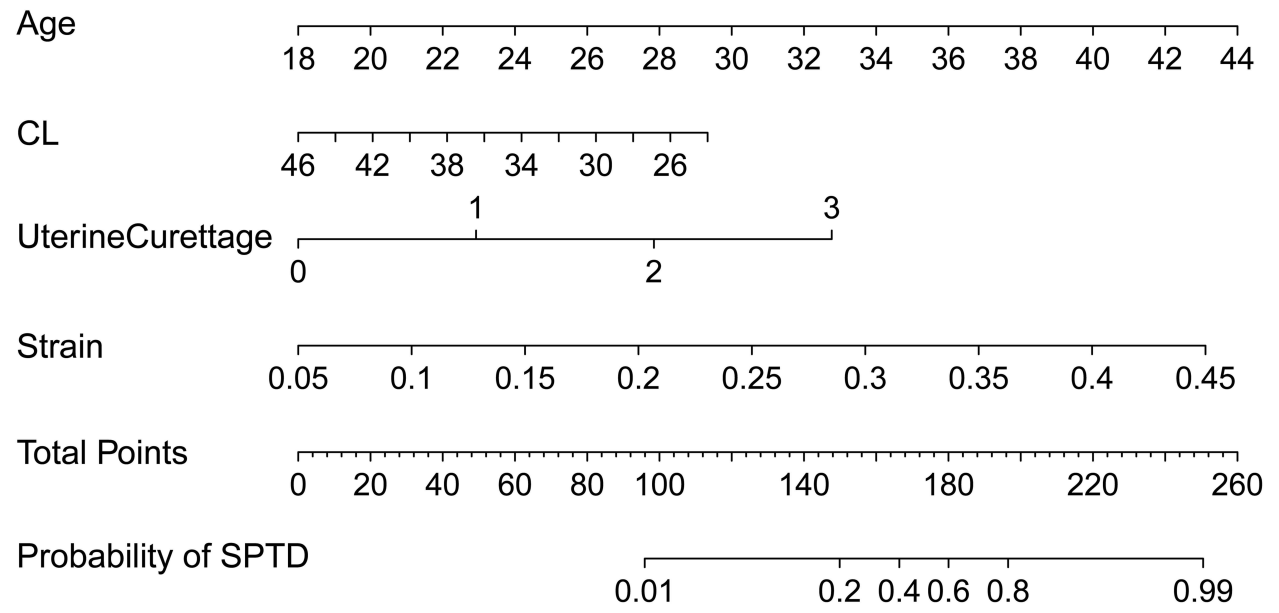

Figure 2 Nomogram predicting the likelihood of SPTD for a pregnant woman in her second trimester. Abbreviations: SPTD, spontaneous preterm delivery; $\mathrm{CL}$, cervical length; Al, anterior lip of internal os. 

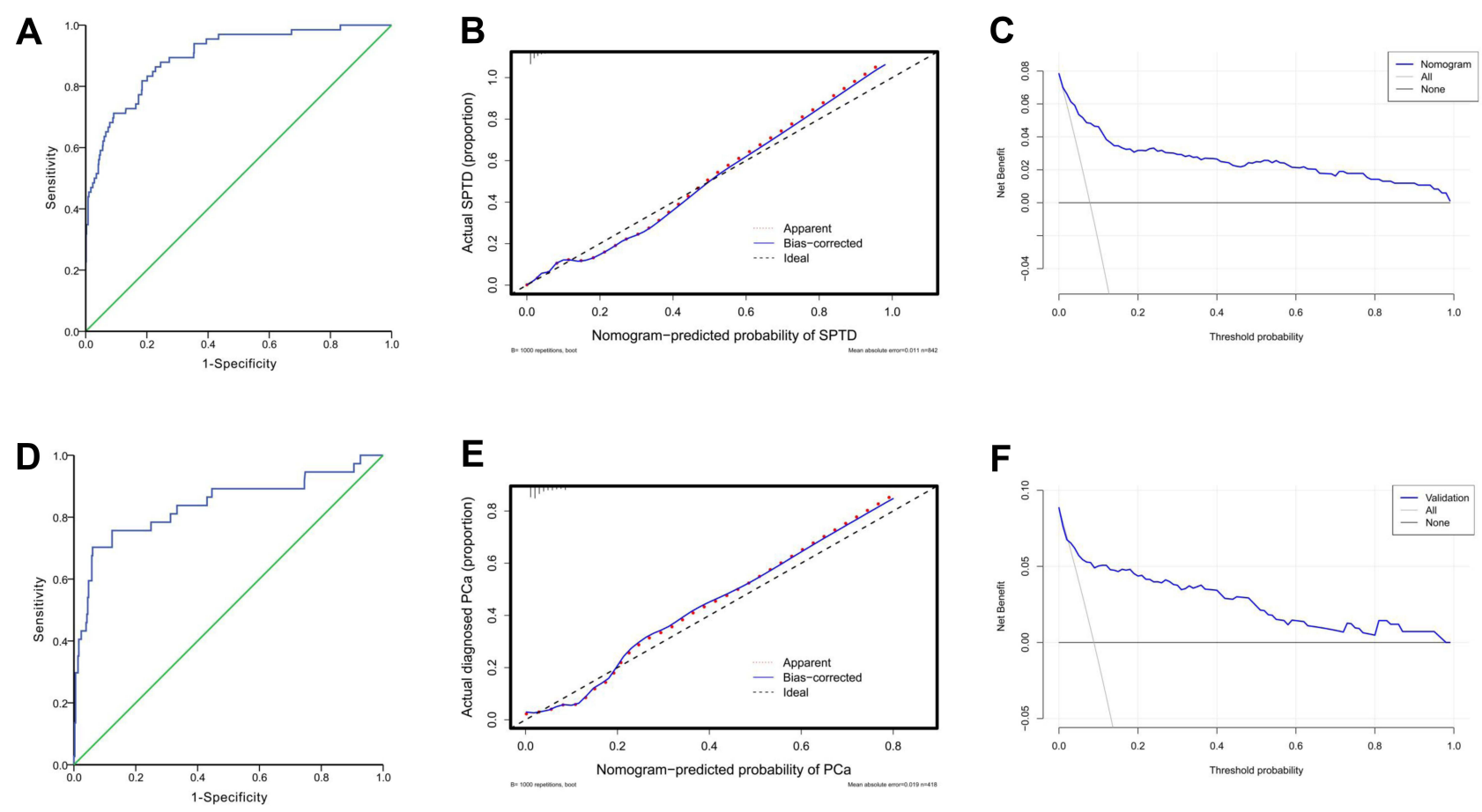

Figure 3 Nomogram verification using training and validation cohorts (A-C for training cohort and D-F for validation cohort). The AUCs of ROC are 0.898 (A) and 0.839 (D). Both calibration plots (B and E) show good agreement. The DCA plots ( $\mathbf{C}$ and $\mathbf{F}$ ) suggest that the probability of SPTD predicted by the nomogram yield net benefit in both cohorts.

Abbreviations: SPTD, spontaneous preterm delivery; ROC, receiver operating characteristic curve; AUC, area under the curve; and DCA, decision curve analysis.

Preterm birth is a major cause of perinatal illness and mortality, but the early prediction is challenging. The use of transvaginal ultrasonography to check $\mathrm{CL}$ as a means to detect a potentially high-risk population is now routinely suggested. However, the incidence of SPTD in pregnant women with CL $\geq 25 \mathrm{~mm}$ is underestimated if applying a cut-off value of $25 \mathrm{~mm}$ in the second trimester, which limits the application of CL in predicting SPTD, especially for low-risk women with normal CL. ${ }^{17}$ Elastography has been used to evaluate the cervix, and recent studies on cervical elastography have demonstrated its utility in predicting SPTD and successful labor induction. ${ }^{18-20}$ However, most studies have concentrated on determining the accuracy of cervical elastography in predicting SPTD (alone or in combination with $\mathrm{CL}$ ), rather than proposing a practical approach for screening possible SPTD according to the measured CL and elastic data. ${ }^{21-23}$ Therefore, we developed a nomogram (an easily applicable prediction model) containing CL and elastic data to predict SPTD.

In addition to CL and strain of AI, age and uterine curettage were found to be the independent predictors of SPTD in this investigation. The OR value of maternal age was 1.29 , which was in line with the range reported by Ferrero et al. ${ }^{24}$ Jacobsson et $\mathrm{al}^{25}$ further reported that preterm birth was more common in women $40-44$ years old, and the OR value for preterm birth $<37$ weeks was 1.54 . The number of uterine curettage is recognized as an independent risk factor for preterm birth, because curettage used to treat miscarriage or termination of pregnancy may induce cervical trauma. ${ }^{26}$ It indicated that the number and method of elective termination should be meticulously recorded, and their influence on SPTD should be thoroughly assessed.

Pregnant women were tested for cervical elasticity during 20 to 24 weeks' gestation in our study. This timeframe is chosen because it corresponds to the typical gestational age range at which a second-trimester anomaly scan is conducted, which helps with clinical promotion. After all, repeatedly evaluating cervical elasticity is unrealistic. We observed in this study that the strain of the internal os was lower than the external os. It meant that the internal os was harder, which was consistent with the ex vivo study by Rosado-Mendez et al, ${ }^{27}$ who found that the stiffness within the cervix increased significantly from distal (vagina) to proximal (uterus). The strains of the AI and PI were associated with SPTD, which was in line with the research by Hernandez-Andrade et $\mathrm{al}^{28}{ }^{2}$ who claimed that the strain values in the 
internal cervical os were linked to the likelihood of SPTD. However, only the strain of AI was found to be an independent predictor of SPTD. We believe that the the anterior lip of internal os is closer to the vaginal probe which will directly absorb the pressure from compression or push pulse and reveal genuine tissue strain. This anatomical region supports a widespread hypothesis that the internal os is necessary for pregnancy maintenance and may play a key role in the mechanism of cervical ripening and labor onset. ${ }^{22,29}$

The main advantage of our study is the establishment of a nomogram with good predictive accuracy for identifying women at high risk of SPTD in the asymptomatic, low-risk population. It allows an individualized decision for preterm birth risk assessment. As described in the Result section (35-year-old woman, once curettage, $\mathrm{CL}=30 \mathrm{~mm}$, and strain of $\mathrm{AI}=0.3$ ), she had a $60 \%$ chance of developing SPTD, implying that active intervention to prevent SPTD might be required. Regarding the verification of the nomogram, strong discrimination and calibration were verified and it generated net benefits both in the training and validation cohorts. The deviation from actual probability was slightly larger in the validation cohort. The nomogram might underestimate the risk of SPTD when the predicted probability was $>20 \%$. It suggested that if the predicted probability was in the range of $40-50 \%$, the actual risk of SPTD might be more than $50 \%$. The decision of intervention should be further evaluated.

Two limitations exist in this study. First, the effectiveness of strain elastography using manual compression is dependent on the sonographer. However, despite the fact that there are little data on the safety of shear wave elastography for fetuses, we did not include it in our study due to the inherent danger of transmitting a focused beam so close to the fetus. ${ }^{8,30,31}$ Second, the nomogram only included data from the second trimester of pregnancy which was suitable for clinical application. It's still unclear which weeks of pregnancy are best for detecting SPTD risk. It's also important to consider when interventions would have a major impact on maternal and perinatal outcomes. However, our study is a preliminary attempt to show that the use of nomogram is possible to identify patients threatened with SPTD in the lowrisk population. Further research should focus on the standardization of the strain evaluation and test the accuracy and feasibility of the nomogram with a larger population in a multi-center study.

\section{Conclusion}

The nomogram including data of multimodal transvaginal ultrasound at 20 to 24 weeks' gestation is a reliable prediction model for identifying women with SPTD in the low-risk population. It seems to be a promising approach for SPTD prediction, and future research is warranted.

\section{Data Sharing Statement}

The data during the current study are available from the corresponding author on reasonable request.

\section{Ethical Approval}

Ethical approval for the study was obtained from the ethics committee of Shanghai Pudong New District Zhoupu Hospital approved this study (2021-C-146).

\section{Informed Consent}

Informed consents were obtained from all participants.

\section{Author Contributions}

Lingli Jiang and Lei Peng contributed equally to this work.

Study design: Lingli Jiang, Lei Peng, Miaoling Rong, and Zhou Liu.

Data collection and analysis: Lingli Jiang, Lei Peng, Miaoling Rong, Xiaozhi Liu, and Qinxia Pang.

Supervision: Lingli Jiang, Lei Peng, and Zhou Liu.

Statistics: Lingli Jiang, Lei Peng, Huaping Li, and Ying Wang.

Manuscript writing: Lingli Jiang, Lei Peng, Miaoling Rong, and Zhou Liu.

Manuscript revision: Lingli Jiang, Lei Peng, and Zhou Liu.

Approval of the manuscript: all authors. 
All authors made a significant contribution to the work reported, whether that is in the conception, study design, execution, acquisition of data, analysis and interpretation, or in all these areas; took part in drafting, revising or critically reviewing the article; gave final approval of the version to be published; have agreed on the journal to which the article has been submitted; and agree to be accountable for all aspects of the work.

\section{Funding}

Pudong New Area Science and Technology Development Fund (PKJ2020-Y46).

\section{Disclosure}

The authors declare that they have no conflicts of interest in this work.

\section{References}

1. Liu L, Oza S, Hogan D, et al. Global, regional, and national causes of under-5 mortality in 2000-15: an updated systematic analysis with implications for the sustainable development goals. Lancet. 2016;388(10063):3027-3035. doi:10.1016/s0140-6736(16)31593-8

2. Volozonoka L, Rots D, Kempa I, et al. Genetic landscape of preterm birth due to cervical insufficiency: comprehensive gene analysis and patient next-generation sequencing data interpretation. PLoS One. 2020;15(3):e0230771. doi:10.1371/journal.pone.0230771

3. Lim K, Butt K, Crane JM. No. 257-ultrasonographic cervical length assessment in predicting preterm birth in singleton pregnancies. $J$ Obstet Gynaecol Can. 2018;40(2):e151-e164. doi:10.1016/j.jogc.2017.11.016

4. Iams JD, Goldenberg RL, Meis PJ, et al.; National institute of child health and human development maternal fetal medicine unit network. The length of the cervix and the risk of spontaneous premature delivery. N Engl J Med. 1996;334(9):567-572. doi:10.1056/nejm199602293340904

5. Mella MT, Berghella V. Prediction of preterm birth: cervical sonography. Semin Perinatol. 2009;33(5):317-324. doi:10.1053/j.semperi.2009.06.007

6. Park HS, Kwon H, Kwon JY, et al. Uterine cervical change at term examined using ultrasound elastography: a longitudinal study. J Clin Med. 2020;10(1):75. doi:10.3390/jcm10010075

7. Catley C, Frize M, Walker CR, Petriu DC. Predicting high-risk preterm birth using artificial neural networks. IEEE Trans Inf Technol Biomed. 2006;10(3):540-549. doi:10.1109/titb.2006.872069

8. Fruscalzo A, Mazza E, Feltovich H, Schmitz R. Cervical elastography during pregnancy: a critical review of current approaches with a focus on controversies and limitations. J Med Ultrason. 2016;43(4):493-504. doi:10.1007/s10396-016-0723-z

9. Gesthuysen A, Hammer K, Möllers M, et al. Evaluation of cervical elastography strain pattern to predict preterm birth. Ultraschall Med. 2020;41 (4):397-403. doi:10.1055/a-0865-1711

10. Wang B, Zhang Y, Chen S, et al. Diagnostic accuracy of cervical elastography in predicting preterm delivery: a systematic review and meta-analysis. Medicine. 2019;98(29):e16449. doi:10.1097/md.0000000000016449

11. Hernandez-Andrade E, Maymon E, Luewan S, et al. A soft cervix, categorized by shear-wave elastography, in women with short or with normal cervical length at 18-24 weeks is associated with a higher prevalence of spontaneous preterm delivery. J Perinat Med. 2018;46(5):489-501. doi:10.1515/jpm-2018-0062

12. Köbbing K, Fruscalzo A, Hammer K, et al. Quantitative elastography of the uterine cervix as a predictor of preterm delivery. $J$ Perinatol. 2014;34 (10):774-780. doi:10.1038/jp.2014.87

13. Park HS, Kwon H, Kwak DW, et al. Addition of cervical elastography may increase preterm delivery prediction performance in pregnant women with short cervix: a prospective study. J Korean Med Sci. 2019;34(9):e68. doi:10.3346/jkms.2019.34.e68

14. Song W, Miao DL, Chen L. Nomogram for predicting survival in patients with pancreatic cancer. Onco Targets Ther. $2018 ; 11: 539-545$. doi:10.2147/ott.S154599

15. Ayubi E, Safiri S. Nomogram predicting long-term survival after the diagnosis of intrahepatic recurrence of hepatocellular carcinoma following an initial liver resection: methodological issues. Int J Clin Oncol. 2017;22(4):803-804. doi:10.1007/s10147-017-1127-9

16. Tang LQ, Li CF, Li J, et al. Establishment and validation of prognostic nomograms for endemic nasopharyngeal carcinoma. J Natl Cancer Inst. 2016;108(1):djv291. doi:10.1093/jnci/djv291

17. Lim K, Butt K, Crane JM. SOGC clinical practice guideline. Ultrasonographic cervical length assessment in predicting preterm birth in singleton pregnancies. J Obstet Gynaecol Can. 2011;33(5):486-499. doi:10.1016/s1701-2163(16)34884-8

18. Fruscalzo A, Schmitz R, Klockenbusch W, Steinhard J. Reliability of cervix elastography in the late first and second trimester of pregnancy. Ultraschall Med. 2012;33(7):E101-e107. doi:10.1055/s-0031-1299330

19. Pereira S, Frick AP, Poon LC, Zamprakou A, Nicolaides KH. Successful induction of labor: prediction by preinduction cervical length, angle of progression and cervical elastography. Ultrasound Obstet Gynecol. 2014;44(4):468-475. doi:10.1002/uog.13411

20. Wozniak S, Czuczwar P, Szkodziak P, Milart P, Wozniakowska E, Paszkowski T. Elastography in predicting preterm delivery in asymptomatic, low-risk women: a prospective observational study. BMC Pregnancy Childbirth. 2014;14(1):238. doi:10.1186/1471-2393-14-238

21. Patberg ET, Wells M, Vahanian SA, et al. Use of cervical elastography at 18 to 22 weeks' gestation in the prediction of spontaneous preterm birth. Am J Obstet Gynecol. 2021;225(5):525.e521-525.e529. doi:10.1016/j.ajog.2021.05.017

22. Hernandez-Andrade E, Garcia M, Ahn H, et al. Strain at the internal cervical os assessed with quasi-static elastography is associated with the risk of spontaneous preterm delivery at $\leq 34$ weeks of gestation. $J$ Perinat Med. 2015;43(6):657-666. doi:10.1515/jpm-2014-0382

23. Suthasmalee S, Moungmaithong S. Cervical shear wave elastography as a predictor of preterm delivery during 18-24 weeks of pregnancy. $J$ Obstet Gynaecol Res. 2019;45(11):2158-2168. doi:10.1111/jog.14094

24. Ferrero DM, Larson J, Jacobsson B, et al. Cross-country individual participant analysis of 4.1 million singleton births in 5 countries with very high human development index confirms known associations but provides no biologic explanation for 2/3 of all preterm births. PLoS One. 2016;11(9): e0162506. doi:10.1371/journal.pone.0162506 
25. Jacobsson B, Ladfors L, Milsom I. Advanced maternal age and adverse perinatal outcome. Obstet Gynecol. 2004;104(4):727-733. doi:10.1097/01. AOG.0000140682.63746.be

26. Lemmers M, Verschoor MA, Hooker AB, et al. Dilatation and curettage increases the risk of subsequent preterm birth: a systematic review and meta-analysis. Hum Reprod. 2016;31(1):34-45. doi:10.1093/humrep/dev274

27. Rosado-Mendez IM, Palmeri ML, Drehfal LC, et al. Assessment of structural heterogeneity and viscosity in the cervix using shear wave elasticity imaging: initial results from a rhesus macaque model. Ultrasound Med Biol. 2017;43(4):790-803. doi:10.1016/j.ultrasmedbio.2016.12.006

28. Hernandez-Andrade E, Romero R, Korzeniewski SJ, et al. Cervical strain determined by ultrasound elastography and its association with spontaneous preterm delivery. J Perinat Med. 2014;42(2):159-169. doi:10.1515/jpm-2013-0277

29. Woźniak S, Czuczwar P, Szkodziak P, Wrona W, Paszkowski T. Elastography for predicting preterm delivery in patients with short cervical length at 18-22 weeks of gestation: a prospective observational study. Ginekol Pol. 2015;86(6):442-447. doi:10.17772/gp/2401

30. Kim H, Hwang HS. Elastographic measurement of the cervix during pregnancy: current status and future challenges. Obstet Gynecol Sci. 2017;60 (1):1-7. doi:10.5468/ogs.2017.60.1.1

31. Swiatkowska-Freund M, Preis K. Cervical elastography during pregnancy: clinical perspectives. Int J Womens Health. 2017;9:245-254. doi:10.2147/ijwh.S106321

International Journal of Women's Health

\section{Publish your work in this journal}

The International Journal of Women's Health is an international, peer-reviewed open-access journal publishing original research, reports, editorials, reviews and commentaries on all aspects of women's healthcare including gynecology, obstetrics, and breast cancer. The manuscript management system is completely online and includes a very quick and fair peer-review system, which is all easy to use. Visit http://www. dovepress.com/testimonials.php to read real quotes from published authors.

Submit your manuscript here: https://www.dovepress.com/international-journal-of-womens-health-journal 